\title{
SPECTROSCOPY OF QUEST RR LYRAE VARIABLES: THE NEW VIRGO STELLAR STREAM Sonia Duffau, ${ }^{1}$ Robert Zinn, ${ }^{2}$ A. Katherina Vivas, ${ }^{3}$ Giovanni Carraro,,${ }^{1,2,4}$ René A. Méndez, ${ }^{1}$ Rebeccah Winnick, ${ }^{2}$ and Carme Gallart ${ }^{5}$
}

\begin{abstract}
Eighteen RR Lyrae variables (RRLs) that lie in the "12.4 clump" identified by the Quasar Equatorial Survey Team (QUEST) have been observed spectroscopically to measure their radial velocities and metal abundances. Ten blue horizontal branch (BHB) stars identified by the Sloan Digital Sky Survey (SDSS) were added to this sample. Six of the nine stars in the densest region of the clump have a mean radial velocity in the Galactic rest frame $\left(V_{\mathrm{gsr}}\right)$ of 99.8 and $\sigma=17.3 \mathrm{~km} \mathrm{~s}^{-1}$, which is slightly smaller than the average error of the measurements. The whole sample contains eight RRLs and five BHB stars that have values of $V_{\mathrm{gsr}}$ suggesting membership in this stream. For seven of these RRLs, the measurements of $[\mathrm{Fe} / \mathrm{H}]$, which have an internal precision of $0.08 \mathrm{dex}$, yield $\langle[\mathrm{Fe} / \mathrm{H}]\rangle=-1.86$ and $\sigma=0.40$. These values suggest that the stream is a tidally disrupted dwarf spheroidal galaxy of low luminosity. Photometry from the database of the SDSS indicates that this stream covers at least $106 \mathrm{deg}^{2}$ of the sky in the constellation Virgo. The name Virgo stellar stream is suggested.
\end{abstract}

Subject headings: Galaxy: halo — Galaxy: kinematics and dynamics — Galaxy: structure stars: variables: other

\section{INTRODUCTION}

A cornerstone of the popular hierarchical picture of galaxy formation is the growth of galaxies with time through multiple mergers. For example, simulations of this process in the framework of $\Lambda$ CDM cosmology by Bullock \& Johnston (2004) suggest that a large galaxy such as the Milky Way experienced $\sim 100$ mergers with dwarf satellite galaxies, and most of them occurred in the first few billion years after the big bang. The advent of large-scale surveys of the Galactic halo during the past decade has provided conclusive proof of comoving groups of stars (stellar streams) exhibiting the kinematic and stellar properties of tidally destroyed dwarf galaxies. The most clear-cut of these are the streams from the Sagittarius (Sgr) dwarf spheroidal (dSph) galaxy and the Monoceros stream (Newberg et al. 2002; Ibata et al. 2003), but a few other less conspicuous, and more uncertain, have been reported. It is also clear that other galaxies, most notably M31 (Ibata et al. 2001), have experienced multiple mergers. The question is not whether mergers occurred but whether or not the number of mergers and their properties match the predictions of the hierarchical picture.

In this Letter, we report spectroscopic observations of the " 12 h.4 clump" of RRLs that was first revealed as an overdensity in the QUEST RRL survey (Vivas et al. 2001; Vivas 2002; Vivas \& Zinn 2003; Zinn et al. 2004; Ivezić et al. 2005) and later in the SDSS as an excess of F-type main-sequence (MS) stars (Newberg et al. 2002). The 12.4 clump is roughly centered at right ascension $12^{\mathrm{h}} 4\left(186^{\circ}\right)$, spans the right ascension range of $\sim 175^{\circ}-200^{\circ}$, the $-2.3-0.0$ range in declination of the QUEST survey, and the Galactic longitude and latitude ranges of $\sim 279^{\circ}-$ $317^{\circ}$ and $\sim 60^{\circ}-63^{\circ}$, respectively. These RRLs have mean $V$ mag-

\footnotetext{
${ }^{1}$ Departamento de Astronomía, Universidad de Chile, Casilla 36-D, Santiago, Chile; sduffau@das.uchile.cl,rmendez@das.uchile.cl, gcarraro@das.uchile.cl.

${ }^{2}$ Department of Astronomy, Yale University, P.O. Box 208101, New Haven, CT 06520-8101; zinn@astro.yale.edu,winnick@astro.yale.edu.

${ }^{3}$ Centro de Investigaciones de Astronomía, Apartado Postal 264, Mérida 5101-A, Venezuela; akvivas@cida.ve.

${ }^{4}$ ANDES fellow, on leave from Dipartimento di Astronomia, Università di Padova, Vicolo Osservatorio 2, I-35122, Padova, Italy.

${ }^{5}$ Instituto de Astrofísica de Canarias, Calle Vía Láctea, E-38200, La Laguna, Tenerife, Spain; carme@iac.es.
}

nitudes, corrected for extinction, equal to $16.9 \pm 0.2$, which with $M_{V}=0.55$ for RRLs, yields a distance from the Sun $\left(r_{\odot}\right)$ of $\sim 19 \mathrm{kpc}$. Speculation on the origin of this clump has centered on the possibility that it is part of the streams of stars from the Sgr dSph galaxy (Majewski et al. 2003; Martínez-Delgado et al. 2004) or the stream from a now extinct dSph galaxy (Vivas \& Zinn 2003).

\section{OBSERVATIONS}

Our campaign to obtain spectroscopic observations of the RRLs in the $12^{\mathrm{h}} 4$ clump began in 2001, but only very modest success was obtained until 2005 because of poor weather and instrument malfunction. Eighteen RRLs have now been observed (QUEST stars 177, 182, 189, 191, 195, 196, 199, 200, 205, 209, $210,224,225,226,233,236,237,261$ ), and 12 have been observed more than once. With the European Southern Observatory $1.5 \mathrm{~m}$ and the Clay Magellan $6.5 \mathrm{~m}$ telescopes, we obtained spectra centered at $4500 \AA$ with resolutions of 3.1 and $4.3 \AA$, respectively. With the WIYN telescope, we obtained spectra centered at $8550 \AA$ with a resolution of $2.9 \AA$. Comparison lamp exposures were taken before and after each stellar one. Stars from Layden (1994) were also observed to serve as standard stars for radial velocity and for his pseudoequivalent width system. With the blue spectra, we cross-correlated each target spectrum with $\sim 10$ spectra of several radial velocity standards of similar spectral type. With the red spectra, we fitted Gaussian line profiles to unblended Paschen lines of hydrogen. To measure the systemic velocity, we fitted the radial velocity curve of $\mathrm{X}$ Arietis to the type $a b$ variables (see Vivas et al. 2005), and for the type $c$ stars (191 and 224) we fitted a template that we constructed from velocity curves of T Sex and DH Peg. The errors in velocities were calculated taking into account the uncertainties in the phases of the observations, the template velocity curves, and the fits. These errors range from 8 to $22 \mathrm{~km} \mathrm{~s}^{-1}$, with a mean value of $17 \mathrm{~km} \mathrm{~s}^{-1}$. Because the RRLs are spread out over $\sim 20^{\circ}$ in right ascension, the Sun's peculiar motion and the motion of the local standard of rest contribute different amounts to their radial velocities. We have therefore removed these effects and base the following analysis on the Galactic standard of rest velocity $\left(V_{\mathrm{gsr}}\right)$, which is the radial velocity mea- 


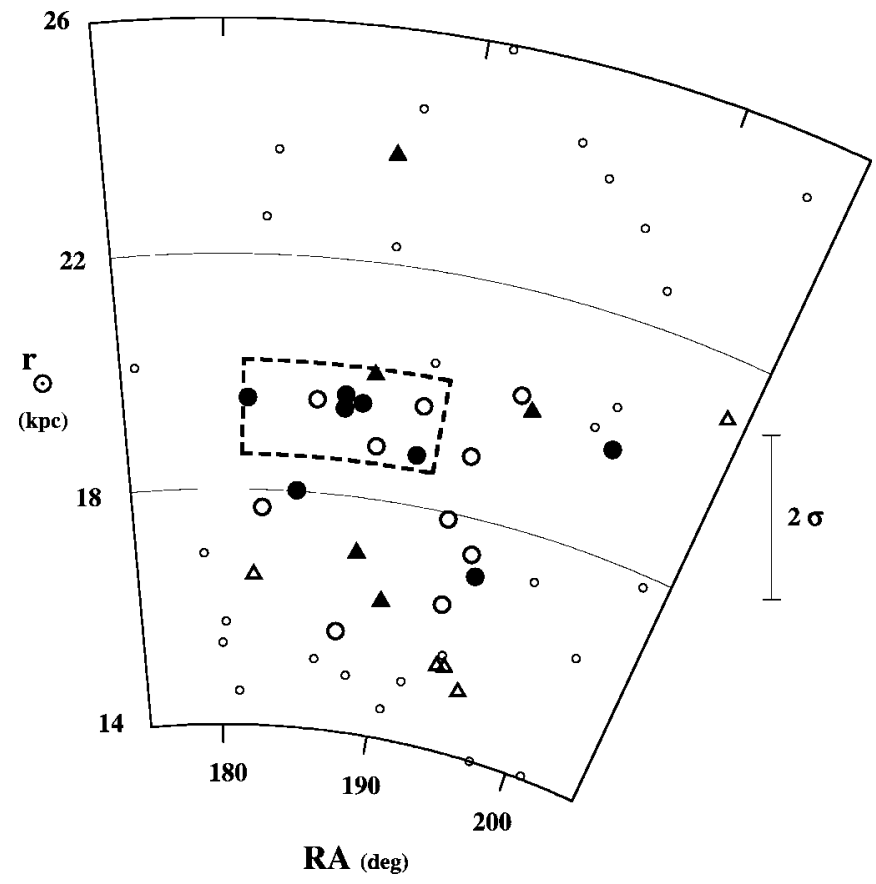

FIG. 1.-Distance from the Sun $\left(r_{\odot}\right)$ plotted against right ascension (RA) for the RRLs in the QUEST survey (circles) and for the BHB stars (triangles) discovered by Sirko et al. (2004a). Large circles depict the RRLs that were observed spectroscopically. Filled symbols depict the RRLs and BHB stars that are probable members of the Virgo stellar stream. The dashed lines enclose the central region (see text). The errors in $r_{\odot}$ scale with distance, and the plotted error bar is appropriate for RRLs at $20 \mathrm{kpc}$. The errors for the BHB stars are roughly a factor of 1.4 larger.

sured by an observer at the Sun who is at rest with the Galactic center. To measure $[\mathrm{Fe} / \mathrm{H}]$ from the blue spectra of the type $a b$ variables, we followed closely Layden's variation of the Preston $\Delta S$ technique (Layden 1994), which cannot be applied to red spectra or to type $c$ stars. The average $\sigma_{[\mathrm{Fe} / \mathrm{H}]}$ for stars observed more than once is $0.08 \mathrm{dex}$, which we consider the internal precision obtained with only one spectrogram (the external error is about \pm 0.15 dex; Layden 1994).

To this sample of RRLs, we added 10 BHB stars that were discovered by Sirko et al. (2004a) in the right ascension range $175^{\circ}-205^{\circ}$, the declination range $-2.5-0.0$, and the $r_{\odot}$ range 15-25 kpc. These stars occupy lines $22,23,28,35,38,41$, 112, 121, 126, and 774 in Table 3 of Sirko et al. (2004a). We have adopted their values of $V_{\text {gsr }}\left(V_{\text {los }}\right.$ in their nomenclature) and also their values of $r_{\odot}$, which are on essentially the same distance scale as the RRLs. Sirko et al. (2004a) estimated that their velocities have an average $\sigma$ of about $26 \mathrm{~km} \mathrm{~s}^{-1}$.

\section{THE KINEMATIC SIGNATURE OF THE STREAM}

In Figure 1, we have plotted our selection of RRLs and BHB stars together with the other QUEST RRLs in the region $175^{\circ}<$ R.A. $<205^{\circ}$ and $14 \mathrm{kpc}<r_{\odot}<26 \mathrm{kpc}$, which encompasses the $12^{\mathrm{h}} .4$ clump. A very tight configuration of RRLs exists at R.A. $\sim 186^{\circ}$ and $r_{\odot} \sim 19.6 \mathrm{kpc}$, and we have drawn a box around this "central region" in Figure 1. We drew this box so as to include a reasonable sample $\left(\sim \frac{1}{3}\right)$ of the total number of stars that had been observed spectroscopically and none that had not been observed. The model of the Galactic halo that Vivas \& Zinn (2003) found was a good match to the distribution of type $a b$ RRLs outside the densest regions in the QUEST catalog predicts that this box should contain only 0.8 of a type $a b$ variable. Since the type $c$ to $a b$ ratio ranges from $\sim 0.2$ to $\sim 0.8$ in

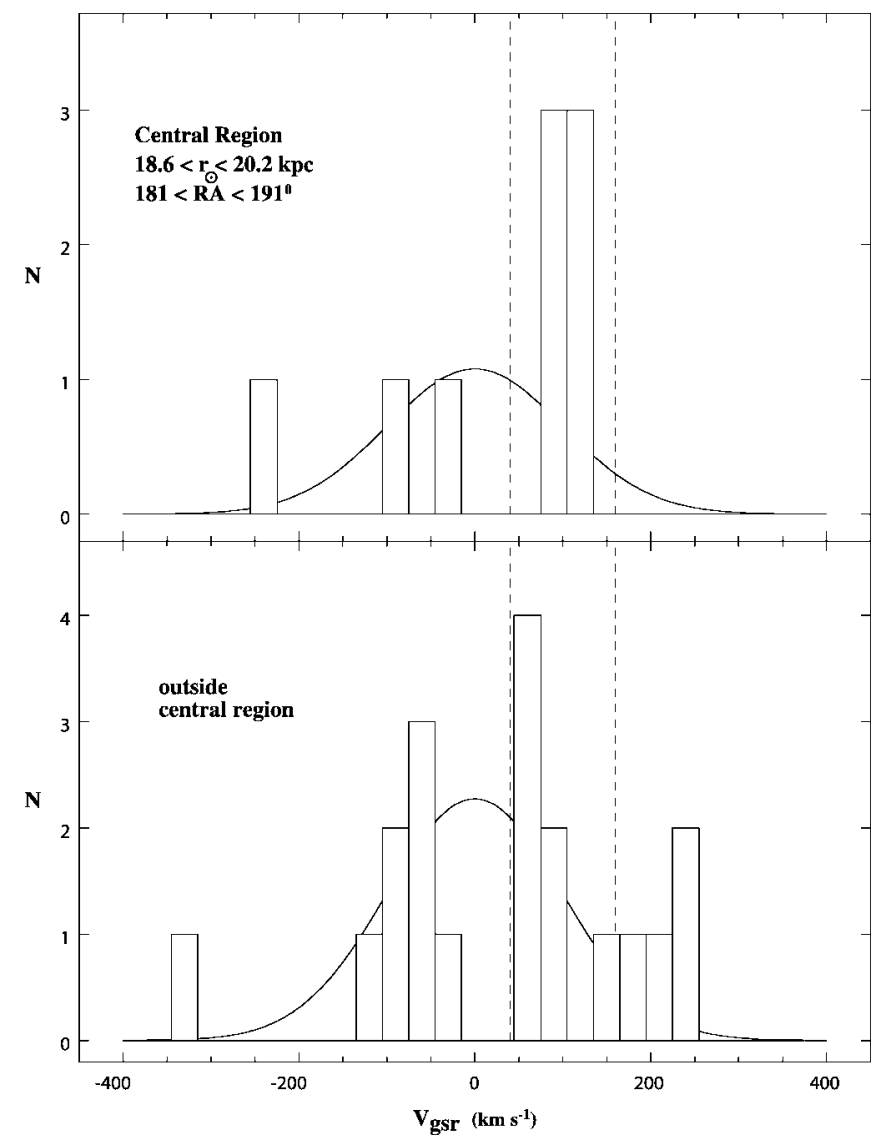

FIG. 2.-Histograms of the $V_{\text {gsr }}$ values of the stars inside (top) and outside (bottom) the central region. Random samples of halo stars that contain the same number of stars are expected to have the normal distributions (solid lines).

globular clusters, depending on Oosterhoff class (Smith 1995), the box is expected to contain $\sim 1-1.4$ RRLs of all types. The observed number of eight RRLs, seven type $a b$ and one type $c$, is clearly a large excess over the expected number. Expanding the box by either reducing the lower limit on $r_{\odot}$ by $\sim 2 \mathrm{kpc}$ or by increasing the upper right ascension limit by $\sim 5^{\circ}$ produces only a little dilution of the kinematic signature of the stream.

In the top panel of Figure 2, we show the histogram of the $V_{\mathrm{gsr}}$ values of the nine stars in the box in Figure 1. The bins are $30 \mathrm{~km} \mathrm{~s}^{-1}$ wide, which is slightly larger than the $1 \sigma$ errors of the stars with least precise measurements. Also plotted is a normal distribution for $\left\langle V_{\mathrm{gsr}}\right\rangle=0$ and $\sigma=100 \mathrm{~km} \mathrm{~s}^{-1}$. A random selection of halo stars is expected to a have a normal distribution (e.g., Harding et al. 2001) and values of $\left\langle V_{\mathrm{gsr}}\right\rangle$ and $\sigma$ close to these values (Sirko et al. 2004b; Brown et al. 2005). Harding et al. (2001) recommended the Shapiro \& Wilk (1965) statistical test of normality as a criterion to apply to halo fields that may harbor stellar streams. According to this test, the $V_{\mathrm{gsr}}$ values of the nine stars are not normally distributed ( $>98 \%$ confidence), and this test is completely independent of any choices for the mean or $\sigma$ of the normal distribution. Six of the nine stars have very closely clustered values of $V_{\mathrm{gsr}}$, $\left\langle V_{\mathrm{gsr}}\right\rangle=99.8$, and remarkably $\sigma=17.3 \mathrm{~km} \mathrm{~s}^{-1}$, which is smaller than the average error $\left(18.8 \mathrm{~km} \mathrm{~s}^{-1}\right)$ of the velocity for these stars. This tight velocity distribution in a volume of space where there is clearly an excess of RRLs is unequivocal evidence for a stellar stream. We suggest that it be called the Virgo stellar stream (VSS) after the constellation in which it is found.

We do not expect to see such a narrow peak in $V_{\mathrm{gsr}}$ as the 
sample is expanded because our measuring errors are substantial; the stream probably has some intrinsic dispersion in velocity and perhaps also a gradient in $\left\langle V_{\mathrm{gsr}}\right\rangle$ with position. Therefore, to investigate the size of the stream, we consider as a possible member any star whose $V_{\mathrm{gsr}}$ lies within $\pm 60 \mathrm{~km} \mathrm{~s}^{-1}$ of the $\left\langle V_{\mathrm{gsr}}\right\rangle$ of the central region (i.e., $40 \mathrm{~km} \mathrm{~s}^{-1} \leq V_{\mathrm{gsr}} \leq 160 \mathrm{~km} \mathrm{~s}^{-1}$, between the vertical dashed lines in Fig. 2). In terms of the measuring errors, this range corresponds to $\pm 2.3 \sigma$ and $\pm 3.6 \sigma$ for the BHB stars and RRLs, respectively. Consequently, in absence of a sizable velocity dispersion and/or gradient, this range is likely to include all the members.

The histogram of the values of $V_{\mathrm{gsr}}$ for the stars lying outside of the central region is shown in the bottom panel of Figure 2. The kinematic signature is much diluted, but it is not completely absent, for there is an excess of stars in the $V_{\mathrm{gsr}}$ range expected of members. Several of these stars have lower values of $V_{\mathrm{gsr}}$ than the members in the central region. This could be due to a velocity gradient, but it is also consistent with the measuring errors and the contamination by nonmembers, which are more likely to have small values of $V_{\mathrm{gsr}}$ than larger ones. We have plotted as solid symbols in Figure 1 the 13 stars $^{6}$ in the whole sample that meet the $V_{\mathrm{gsr}}$ criterion for membership. These stars span large ranges in right ascension and $r_{\odot}$, suggesting that the VSS is both large and diffuse. Two of these stars stand out as the most likely nonmembers: BHB star 23, which lies $\sim 4 \mathrm{kpc}$ more distant than the major concentration, and RRL 261, which is the RRL lying farthest to the east and is also the star in this sample with the largest value of $V_{\mathrm{gsr}}$. The removal of these two stars from the sample changes $\left\langle V_{\mathrm{gsr}}\right\rangle$ and $\sigma$ from 85.4 and $31.4 \mathrm{~km} \mathrm{~s}^{-1}$, respectively, to 83.2 and $24.5 \mathrm{~km} \mathrm{~s}^{-1}$. Since this last $\sigma$ is not much larger than the average measuring error for this sample $\left(20.3 \mathrm{~km} \mathrm{~s}^{-1}\right)$, more precise measurements are needed to refine the membership criterion and to be certain that the velocity dispersion has been resolved. Our future measurements of RRLs should indicate whether the VSS extends in the directions suggested by BHB star 23 and RRL 261.

\section{THE SIGNATURE IN THE LUMINOSITY FUNCTION}

Newberg et al. (2002) discovered an excess of F-type MS stars in the SDSS in the direction to the $12^{\mathrm{h}} .4$ clump and at the appropriate magnitude to be related to the RRLs (feature S297+63-20.0). In Figure 1 of Newberg et al. (2002), the globular cluster Pal 5 appears as a streak pointing radially toward the Sun. This is a sign that this method, which is excellent for finding halo substructure, may not locate precisely an old stellar population. We have therefore examined the SDSS photometry for evidence of the upturn in the luminosity function (LF) of an old stellar population that occurs near the magnitude where the subgiant branch (SGB) and the MS merge (see also Martínez-Delgado et al. 2002).

The method is illustrated in the top three panels of Figure 3. The top left panel shows the color magnitude diagram (CMD) of $2^{\circ} \times 2^{\circ}$ centered on the globular cluster Pal 5. The cluster and its tidal tails occupy only a fraction of this field. The top middle panel is the CMD of a control field of equal area. The top right panel shows the LFs of the Pal 5 field and the control field. These functions were constructed using the color indices c1 and c2 as defined by Odenkirchen et al. (2001) and their filtering technique, which minimizes the contamination by poor measurements and by field stars. While the presence of Pal 5 is clearly detectable in the CMD itself, it is very evident in the

\footnotetext{
${ }^{6}$ Eight of them are RRLs (177, 189, 195, 196, 199, 210, 237, and 261), and five are BHB stars $(22,23,28,38,121$ in Table 3 of Sirko et al. 2004a).
}

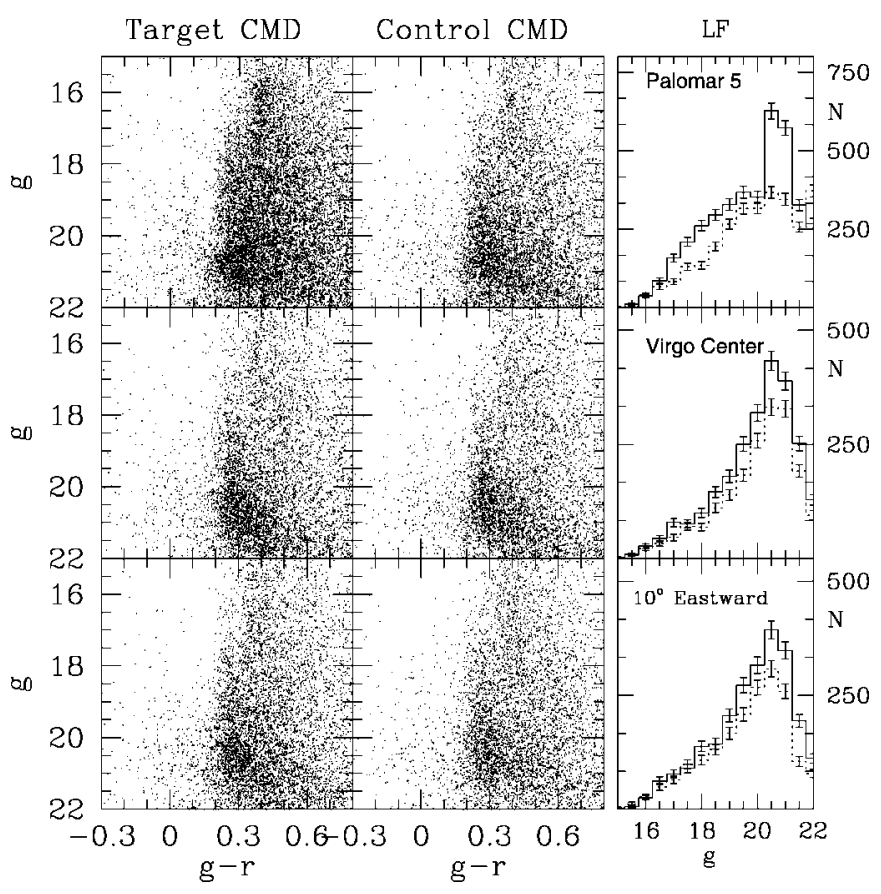

FIG. 3.-Left panels: CMDs from SDSS photometry for $2^{\circ} \times 2^{\circ}$ fields that include (top) the globular cluster Palomar 5, (middle) the center of the VSS $\left(186^{\circ}<\right.$ R.A. $<188^{\circ},-2^{\circ}<$ decl. $\left.<0^{\circ}\right)$, and (bottom) an eastern field of the VSS $\left(196^{\circ}<\right.$ R.A. $<198^{\circ},-2^{\circ}<$ decl. $\left.<0^{\circ}\right)$. In the middle panels, we show the CMDs for control fields of equal size that were offset by $10^{\circ}$ or more. In the right panels, the luminosity functions of the object field (solid line) and the control field (dashed line) are plotted as histograms (Poisson error bars).

right panel as a sharp increase in the number of stars at $g \sim$ 20.25, which is roughly the magnitude of the SGB (the MS turnoff is $\sim 0.5$ fainter; Smith et al. 1986).

The middle panels in Figure 3 show the CMDs and the LFs of the central part of the VSS and a comparison field. The three RRLs in this VSS field have $\langle V\rangle=17.09$, whereas the five RRLs in Pal 5 have $\langle V\rangle=17.44$ (Vivas et al. 2004). If VSS resembles Pal 5, a sharp upturn in the LF is expected at $g \sim$ 19.9. While the VSS field shows an excess of stars over its comparison field at this magnitude, its LF clearly does not mimic that of Pal 5. However, Pal 5 is a moderately metal-rich globular cluster $([\mathrm{Fe} / \mathrm{H}]=-1.47$; Zinn \& West 1984), and it has the combination of age and metallicity that produces a nearly horizontal SGB and consequently a very sharp upturn in the LF. The seven RRLs in the VSS with measurements of $[\mathrm{Fe} / \mathrm{H}]$ have a mean value of -1.86 , which suggests that a more metal-poor globular cluster than Pal 5 would be a better comparison object. The LF constructed by Zaggia et al. (1997) for the globular cluster M55 $([\mathrm{Fe} / \mathrm{H}]=-1.82$; Zinn $\&$ West 1984$)$ shows a more gradual increase because its SGB is more steeply inclined in the CMD than Pal 5's. If the LF of the VSS resembles that of M55, a relatively gradual climb in the number of stars is expected to start at $M_{V} \sim 3.0$, or $g \sim 19.5$. Since the LF in the middle panel of Figure 3 shows this behavior, we believe the excess of F-type MS stars first recognized in the SDSS data by Newberg et al. (2002) is indeed the MS of the VSS. The bottom panels show the CMDs and the LFs for a field $10^{\circ}$ to the east of the central one, where the VSS appears to be present but not as strongly as in the central one.

Using this technique with the SDSS photometry, we searched for the VSS in $272 \mathrm{deg}^{2}$, between $176^{\circ} \leq$ R.A. $\leq 210^{\circ}$ and $-4^{\circ} \leq$ decl. $\leq+4^{\circ}$. We considered that the VSS was detected if in the range $19.5<g<20.5$ the number of stars in the target 
field was consistently greater than the number in the control field by an amount larger than the combined Poisson errors. The control and target fields had similar Galactic latitudes but were offset from each other by $10^{\circ}$ or more. Figure 4 shows the region of the sky $\left(\sim 106 \mathrm{deg}^{2}\right)$ where the VSS was detected. Toward the east, the VSS becomes less prominent due to the progressive increase of the field population. The northern part of the VSS $\left(0^{\circ}<\right.$ decl. $\left.<2^{\circ}\right)$ has a somewhat brighter upturn in the LF than the southern portion $\left(-4^{\circ}<\right.$ decl. $\left.<-2^{\circ}\right)$, which indicates that it is closer to the Sun.

\section{DISCUSSION}

The previous suggestions (Majewski et al. 2003; MartínezDelgado et al. 2004) that the $12^{\mathrm{h}} .4$ clump of RRLs may be part of the streams from the Sgr dSph are inconsistent with our observations. The model of the Sgr streams by Martínez-Delgado et al. (2004) predicts a very low density of Sgr stars in this volume of space, which is in conflict with the concentration seen in Figure 1. The models by Law et al. (2005) that assume either a spherical or an oblate shape for the Milky Way's dark matter halo predict more significant numbers of Sgr stars in this volume but with $\left\langle V_{\mathrm{gsr}}\right\rangle \sim-180$ and $\sim-260 \mathrm{~km} \mathrm{~s}^{-1}$, respectively. These values are completely incompatible with the $\left\langle V_{\mathrm{gsr}}\right\rangle$ of the VSS ( +83 or $+100 \mathrm{~km} \mathrm{~s}^{-1}$, depending on sample selection). According to the prolate model of Law et al. (2005), this region should not contain debris from Sgr.

Based on our measurements for seven type $a b$ RRLs that have values of $V_{\mathrm{gsr}}$ that are consistent with membership, the VSS has $\langle[\mathrm{Fe} / \mathrm{H}]\rangle=-1.86$ and $\sigma=0.40$. Because this dispersion is several times the average of the $\sigma_{[\mathrm{Fe} / \mathrm{H}]}$ values $(0.08)$, we conclude that the VSS has a significant range in $[\mathrm{Fe} / \mathrm{H}]$. A range of this magnitude is characteristic of all the dSph satellite galaxies of the Milky Way but not of the vast majority of globular clusters. The VSS is probably the debris of a disrupted dSph galaxy. There is no sign of a significant intermediate-age population in the CMDs from the SDSS data (Newberg et al. 2002 and the ones we produced), and the lists of halo carbon stars (Totten \& Irwin 1998; Mauron et al. 2004, 2005) do not contain any that are consistent with membership in the VSS. Thus, the progenitor of the VSS appears to have been a system dominated by its very old stellar population. These properties suggest that of the extant $\mathrm{dSph}$ galaxies, the VSS may most closely resemble the ones of

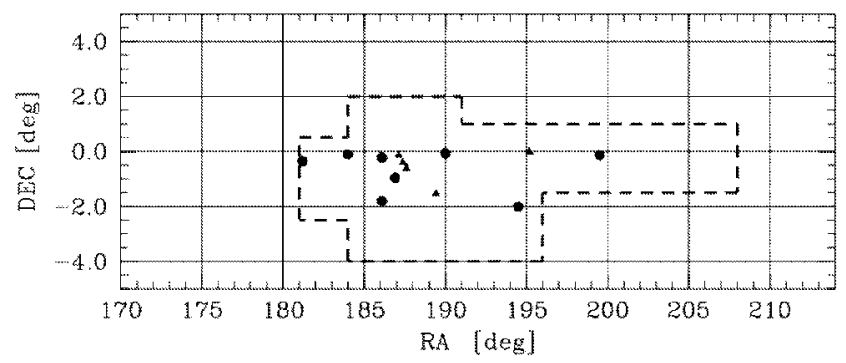

FIG. 4.- The dashed lines enclose the region of the sky where we found an excess of stars compared to the control fields in the interval $\sim 19.5<g<$ 20.5. The RRLs and BHB stars that are possibly members of the Virgo stellar stream (i.e., the stars within the dashed vertical lines in Fig. 2 and solid symbols in Fig. 1) are plotted as circles and triangles, respectively.

the lowest luminosity, e.g., Ursa Minor, Draco, and Sextans (Mateo 1998).

Finally, our measurements have also revealed a few other interesting features. Ignoring the VSS stars, there are some smaller groups of stars that have positions and values of $\left\langle V_{\mathrm{gsr}}\right\rangle$ that suggest possible membership in the same "moving group" (e.g., RRL 226, 233, and BHB 35; RRL 224, 225, 236, and BHB 126). Furthermore, if we consider together the 15 stars that are not members of the VSS and the two that have low probabilities of membership (RRL 261 and BHB 23), we find $\left\langle V_{\mathrm{gsr}}\right\rangle=-9.1$ and $\sigma=163.7 \mathrm{~km} \mathrm{~s}^{-1}$. This value of $\sigma$ is remarkably large, and it is inconsistent at above the $95 \%$ confidence level ( $F$-test) with the value of $101.6 \mathrm{~km} \mathrm{~s}^{-1}$ that Sirko et al. (2004b) found from their sample of 1170 BHB stars with median $r_{\odot} \sim 25 \mathrm{kpc}$. The origin of this kinematically hot distribution, the possibility of other streams besides the VSS, and the relationship of the VSS to the large feature recently reported by Jurić et al. (2005) in Virgo are being investigated as we obtain additional spectroscopy of QUEST RRLs.

This research is part of a joint project between the Universidad de Chile and Yale University, which is partially funded by the Fundación Andes. We acknowledge funding from the following institutions and grants: NSF AST 00-98428, NSF AST 05-07364, Fundación Andes C-13798, MECESUP UCH118, and FONDAP 15010003.

\section{REFERENCES}

Brown, W. R., Geller, M. J., Kenyon, S. J., Kurtz, M. J., Allende Prieto, C., Beers, T. C., \& Wilhelm, R. 2005, AJ, 130, 1097

Bullock, J. S., \& Johnston, K. V. 2004, in ASP Conf. Ser. 327, Satellites and Tidal Streams, ed. F. Prada, D. Martínez-Delgado, \& T. J. Mahoney (San Francisco: ASP), 80

Harding, P., Morrison, H. L., Olszewski, E. W., Arabadjis, J., Mateo, M., Dohm-Palmer, R. C., Freeman, K. C., \& Norris, J. E. 2001, AJ, 122, 1397 Ibata, R., Irwin, M., Lewis, G., Ferguson, A. M. N., \& Tanvir, N. 2001, Nature, 412,49

. 2003, MNRAS, 340, L21

Ivezić, Ž., Vivas, A. K., Lupton, R. H., \& Zinn, R. 2005, AJ, 129, 1096

Jurić, M., et al. 2005, preprint (astro-ph/0510520)

Law, D. R., Johnston, K. V., \& Majewski, S. R. 2005, ApJ, 619, 807

Layden, A. C. 1994, AJ, 108, 1016

Majewski, S. R., Skrutskie, M. F., Weinberg, M. D., \& Ostheimer, J. C. 2003, ApJ, 599, 1082

Martínez-Delgado, D., Gómez-Flechoso, M. Á., Aparicio, A., \& Carrera, R. 2004, ApJ, 601, 242

Martínez-Delgado, D., Zinn, R., Carrera, R., \& Gallart, C. 2002, ApJ, 573, L19

Mateo, M. 1998, ARA\&A, 36, 435
Mauron, N., Azzopardi, M., Gigoyan, K., \& Kendall, T. R. 2004, A\&A, 418, 77

Mauron, N., Kendall, T. R., \& Gigoyan, K. 2005, A\&A, 438, 867

Newberg, H. J., et al. 2002, ApJ, 569, 245

Odenkirchen, M., et al. 2001, ApJ, 548, L165

Shapiro, S. S., \& Wilk, M. B. 1965, Biometrika, 52, 591

Sirko, E., et al. 2004a, AJ, 127, 899

$$
\text { 2004b, AJ, 127, } 914
$$

Smith, G. H., McClure, R. D., Stetson, P. B., Hesser, J. E., \& Bell, R. A. 1986, AJ, 91, 842

Smith, H. A. 1995, RR Lyrae Stars (Cambridge: Cambridge Univ. Press)

Totten, E. J., \& Irwin, M. J. 1998, MNRAS, 294, 1

Vivas, A. K. 2002, Ph.D. thesis, Yale Univ.

Vivas, A. K., \& Zinn, R. 2003, Mem. Soc. Astron. Italiana, 74, 928

Vivas, A. K., Zinn, R., \& Gallart, C. 2005, AJ, 129, 189

Vivas, A. K., et al. 2001, ApJ, 554, L33 2004, AJ, 127, 1158

Zaggia, S. R., Piotto, G., \& Capaccioli, M. 1997, A\&A, 327, 1004

Zinn, R., Vivas, A. K., Gallart, C., \& Winnick, R. 2004, in ASP Conf. Ser. 327, Satellites and Tidal Streams, ed. F. Prada, D. Martínez-Delgado, \& T. J. Mahoney (San Francisco: ASP), 92

Zinn, R., \& West, M. J. 1984, ApJS, 55, 45 